\title{
Cascading on extragalactic background light (Research Note)
}

\author{
P. d'Avezac ${ }^{1}$, G. Dubus ${ }^{1,2,3}$, and B. Giebels ${ }^{1}$ \\ ${ }^{1}$ Laboratoire Leprince-Ringuet, UMR 7638 CNRS, École Polytechnique, 91128 Palaiseau, France \\ e-mail: Guillaume.Dubus@obs.ujf-grenoble. fr \\ 2 Institut d'Astrophysique de Paris, UMR 7095 CNRS, Université Pierre \& Marie Curie, 98bis Bd. Arago, 75014 Paris, France \\ ${ }^{3}$ Laboratoire d'Astrophysique de Grenoble, UMR 5571 CNRS, Université Joseph Fourier, BP 53, 38041 Grenoble, France
}

Received 8 November 2006 / Accepted 21 April 2007

ABSTRACT

\begin{abstract}
Context. High-energy $\gamma$-rays propagating in the intergalactic medium can interact with background infrared photons to produce $\mathrm{e}^{+} \mathrm{e}^{-}$pairs, resulting in the absorption of the intrinsic $\gamma$-ray spectrum. TeV observations of the distant blazar 1ES 1101-232 were thus recently used to put an upper limit on the infrared extragalactic background light density.

Aims. The created pairs can upscatter background photons to high energies, which in turn may pair produce, thereby initiating a cascade. The pairs diffuse on the extragalactic magnetic field (EMF) and cascade emission has been suggested as a means for measuring its intensity. Limits on the IR background and EMF are reconsidered taking into account cascade emissions.

Methods. The cascade equations are solved numerically. Assuming a power-law intrinsic spectrum, the observed $100 \mathrm{MeV}-100 \mathrm{TeV}$ spectrum is found as a function of the intrinsic spectral index and the intensity of the EMF.

Results. Cascades emit mainly at or below $100 \mathrm{GeV}$. The observed $\mathrm{TeV}$ spectrum appears softer than for pure absorption when cascade emission is taken into account. The upper limit on the IR photon background is found to be robust. Inversely, the intrinsic spectra needed to fit the $\mathrm{TeV}$ data are uncomfortably hard when cascade emission makes a significant contribution to the observed spectrum. An EMF intensity around $10^{-8} \mathrm{nG}$ leads to a characteristic spectral hump in the GLAST band. Higher EMF intensities divert the pairs away from the line-of-sight and the cascade contribution to the spectrum becomes negligible.
\end{abstract}

Key words. radiation mechanisms: non-thermal - galaxies: BL Lacertae objects: individual: 1ES 1101-232 galaxies: intergalactic medium - cosmology: diffuse radiation - gamma rays: observations

\section{Introduction}

The observed very high energy (VHE) spectra of extragalactic sources are attenuated by pair production $(\mathrm{PP})$ on background photon fields. At energies $\epsilon=100 \mathrm{TeV}$, interactions with CMB photons make the universe opaque beyond a few Mpc (Gould \& Schréder 1966). In the 1-10 TeV range, the target photon field is the infrared extragalactic background light (EBL), with an undetermined horizon $z \gtrsim 0.2$ due to uncertainties in the EBL density at optical to UV wavelengths. Conversely, observations of absorbed VHE spectra of blazars can constrain the EBL density at these wavelengths, provided their intrinsic emission is known (Stecker et al. 1992; Biller 1995). Recently, the HESS collaboration used its observations of 1ES 1101-232 $(z=0.186)$, together with a reasonable assumption on the intrinsic spectrum, to estimate a stringent upper limit to the EBL (Aharonian et al. 2006). This estimation did not consider emission from the cascade initiated when the created pairs upscatter EBL photons back to VHE energies (Protheroe 1986; Protheroe \& Stanev 1993; Aharonian et al. 1994; Biller 1995; Aharonian et al. 2002). Cascade emission may make the universe appear more transparent than under the assumption of pure absorption. Inversely, including cascade emission when deconvolving for propagation effects on a given EBL leads to intrinsic spectra different from the pure absorption case.

The impact of this emission on the EBL upper limit, as derived from the 1ES 1101-232 spectrum, is considered here. Electrons may diffuse on the extragalactic magnetic field (EMF), causing their emissions to be lost for the observer (Protheroe 1986; Aharonian et al. 1994; Plaga 1995). The EBL and EMF governing the propagation of the cascade are described in Sect. 2. The cascade equations and numerical method are described in Sect. 3 and applied to the case of 1ES 1101-232 in Sect. 4. The implications on the EBL and EMF limits are set out in Sect. 5.

\section{Extragalactic backgrounds}

In addition to the CMB, the diffuse photon background is constituted of integrated emission from stars (peaking around $2 \mu \mathrm{m}$, see Fig. 1) and heated dust (peaking around $200 \mu \mathrm{m}$ ). The EBL spectral energy distribution is difficult to measure directly because of the strong zodiacal and galactic foregrounds. Lower limits have been set using source counts while SED shapes have been derived from simulations of galaxy formation (Primack 2002; Lagache et al. 2003; Xu et al. 2001). The EBL shape HESS 2006 (Fig. 1) was computed by Primack et al. (1999) and normalised by a factor 0.45 in Aharonian et al. (2006) to account for the $\mathrm{TeV}$ observations of 1ES 1101-232. Primack 2005 refers to an EBL normalised instead to fit the lower limit set by galaxy counts. The Spitzer observations suggest higher fluxes in the $10-1000 \mu \mathrm{m}$ range (Dole et al. 2006). This affects attenuation above $20 \mathrm{TeV}$ but has been verified to have no consequence on the results presented here.

The created pairs can be deflected from the line-of-sight by an extragalactic magnetic field (EMF). Faraday rotation and 


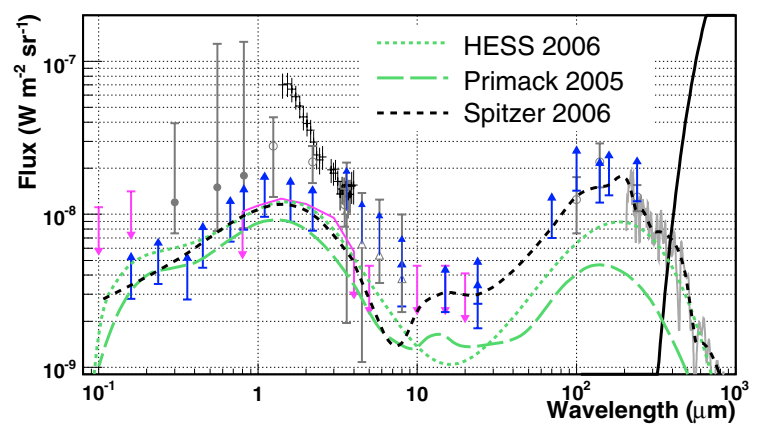

Fig. 1. EBL $+C M B$ photon density (at $z=0$ ) used in this work. The HESS 2006 and Primack 2005 EBL derive from a simulation of galaxy formation (Primack et al. 1999, 2005, respectively). The Spitzer 2006 EBL is a best fit to available observations (Dole et al. 2006, from which the measurements shown here were also taken).

synchrotron emission in radio yield estimates of magnetic fields in galaxies (roughly $>10 \mathrm{nG}$ ), or in clusters $(\leq 0.1-1 \mathrm{nG})$ and even some super- clusters $(\leq \mathrm{nG})$ (Kronberg 1994; Widrow 2002; Vallée 2004). The EMF outside these structures is unconstrained and may be as low as $10^{-19} \mathrm{nG}$ (Fan et al. 2003, and references therein). For such very weak EMFs, the deflection of electrons due to IC interactions is negligible and the cascade occurs along the line-of-sight with a short delay of the secondary emission (Plaga 1995; Cheng \& Cheng 1996; Dai et al. 2002). Diffusion on a stronger EMF creates a halo around $\gamma$-ray sources and isotropizes the cascade emission (Aharonian et al. 1994). This occurs when the gyroradius $R_{\mathrm{L}}$ of the pairs is much lower than their Compton cooling length $C_{\mathrm{IC}}=E(\mathrm{~d} E / \mathrm{d} l)_{\mathrm{IC}}^{-1}$. Since mostly $\mathrm{CMB}$ photons are upscattered, the minimum $B$ required to isotropise pairs of energy $E$ is $3 \times 10^{-6} E_{\mathrm{TeV}}^{2}(1+z)^{4} \mathrm{nG}$. Much of the isotropic re-emission is lost to the observer and the pairs diffuse on a scale $\sim\left(R_{\mathrm{L}} C_{\mathrm{IC}}\right)^{1 / 2}$. For intermediate EMFs, the $\mathrm{TeV}$ electrons in the beamed relativistic jet are deflected by $\sim C_{\mathrm{IC}} / R_{\mathrm{L}}$. Halo sizes $\gtrsim 0.1^{\circ}$ could be resolved by $\gamma$-ray detectors and used to estimate the EMF intensity (Neronov \& Semikoz 2006). Photons in $0.1^{\circ}$ haloes have propagation times varying by $\sim 10^{5}$ years, averaging out any time variability (Fan et al. 2003). In the following, the cascade emission is assumed to be unresolved from the source and delays are not considered. The TeV emission detected by HESS from 1ES 1101-232 appears to be at a low flux level with no significant variability.

\section{Cascade equations}

The cascade is described by a set of two coupled equations involving the photon energy density $n_{\mathrm{P}}(\varepsilon)$ and the electron (positron) energy density $n_{\mathrm{E}}(E)$ :

$$
\begin{aligned}
c \partial_{t} n_{\mathrm{P}}= & -\frac{1}{\lambda_{\mathrm{PP}}} n_{\mathrm{P}}+c_{B} \int_{\varepsilon}^{+\infty} \mathrm{G}_{\mathrm{IC}}(e, \varepsilon) n_{\mathrm{E}}(e) \mathrm{d} e \\
c \partial_{t} n_{\mathrm{E}}= & -\frac{1}{\lambda_{\mathrm{IC}}} n_{\mathrm{E}}+2 \int_{E}^{+\infty} \mathrm{G}_{\mathrm{PP}}(e, E) n_{\varepsilon}(e) \mathrm{d} e \\
& +\int_{E}^{+\infty} \mathrm{G}_{\mathrm{IC}}(e, e-E) n_{\mathrm{E}}(e) \mathrm{d} e .
\end{aligned}
$$

The first term in both equations is the sink term due to PP (Eq. (1)) or IC losses (Eq. (2)). $\lambda_{\mathrm{PP}}$ and $\lambda_{\mathrm{IC}}$ are the mean free path for each interaction. The second term is the source term corresponding to cascade emission (Eq. (1)) or pair creation (Eq. (2), with a factor 2 for the pair). The cascade emission factor $c_{B}$ is 1

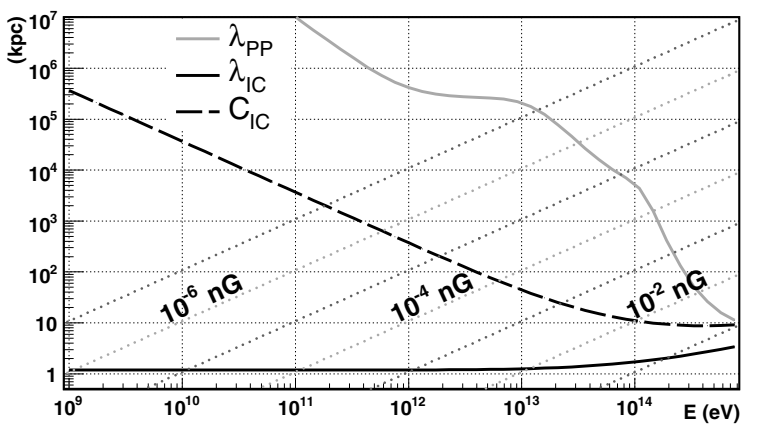

Fig. 2. Mean free path $\lambda_{\mathrm{PP}}$ for PP on the EBL+CMB background as a function of the VHE photon energy. The mean free path $\lambda_{\text {IC }}$ and IC cooling length $C_{\mathrm{IC}}$ of the pairs on the CMB is also shown as function of electron energy. $C_{\mathrm{IC}}$ flattens (then rises) at high energies as the interaction enters the Klein-Nishina regime. The electron then loses its energy in a single interaction $\left(\lambda_{\mathrm{IC}} \approx C_{\mathrm{IC}}\right)$. IC losses of the pairs take place on a small scale compared to the $\gamma$-ray attenuation length for photon energies $<300 \mathrm{TeV}$. The electron gyroradius $R_{\mathrm{L}}$ for various EMF intensities is indicated by dashed diagonal lines. The pairs are expected to be isotropised by the EMF for energies and B intensities such that $R_{\mathrm{L}} \leq C_{\mathrm{IC}}$.

when the EMF is ignored, and approximated to 0 when the electron population is considered isotropised. The pair production term is written in terms of $\mathrm{G}_{\mathrm{PP}}(\varepsilon, E)=\int \partial_{E} \sigma_{\mathrm{PP}}\left(\varepsilon, \varepsilon_{\mathrm{B}}\right) u\left(\varepsilon_{\mathrm{B}}\right) \mathrm{d} \varepsilon_{\mathrm{B}}$, where $\partial_{E} \sigma_{\mathrm{PP}}$ is the differential cross-section and $u$ is the photon background energy density $(\mathrm{EBL}+\mathrm{CMB})$. The IC radiation term $\mathrm{G}_{\mathrm{IC}}(E, \varepsilon)$ is defined similarly. The third term in Eq. (2) reflects IC cooling of electrons from higher energies. All of these terms are functions of $z$.

The integrated cross-sections for PP and IC on isotropic target photons are taken from Gould \& Schréder (1966) and Jones (1967). Analytic expressions of the differential cross- sections derived by Zdziarski (1988) for background densities in the form of blackbodies or power laws are used to calculate $\mathrm{G}_{P P}$ and $\mathrm{G}_{I C}$. The cascade equations are solved numerically by combining $n_{\mathrm{P}}$ and $n_{\mathrm{E}}$ into a single vector $V$ defined on a logarithmic scale of energies $\left(\varepsilon_{0} \zeta^{i}\right)$, from $\varepsilon_{0}=10^{7} \mathrm{eV}$ up to $10^{17} \mathrm{eV}$ in 250 steps (thus $\left.\zeta=\left(10^{17} / 10^{7}\right)^{1 / 250}\right)$. To ensure energy conservation, the integrals on $\mathrm{G}_{\mathrm{PP}}$ and $\mathrm{G}_{\mathrm{IC}}$ are calculated as

$$
\int \mathrm{G}_{\mathrm{IC}}(e, \varepsilon) n_{\mathrm{E}}(e) \mathrm{d} e=\sum_{k} V_{k, E} \int_{\zeta^{-1 / 2}}^{\zeta^{1 / 2}} \frac{\epsilon_{0} \zeta^{k} u \mathrm{G}_{\mathrm{IC}}\left(\epsilon_{0} \zeta^{k} u, \varepsilon\right) \mathrm{d} u}{\zeta^{1 / 2}-\zeta^{-1 / 2}}
$$

The cascade equations may then be rewritten as a matrix $\mathbf{P}$ acting on the vector $V: V(t+\delta t)=\exp (\delta t \mathbf{P}) V(t)$ (exp is developed to the 4 th order in $\delta t)$. The terms in $\mathbf{P}$ are of the order of $\lambda_{\mathrm{IC}}^{-1}$ or less, hence it is enough to take steps of size $c \delta t=0.1 \mathrm{kpc}$, updating the matrix $\mathbf{P}(z)$ every $\delta z=0.001$ with $\mathrm{d} z=H_{0}(1+z)\left[\Omega_{M}(1+\right.$ $\left.z)^{3}+\Omega_{\Lambda}+\left(1-\Omega_{M}-\Omega_{\Lambda}\right)(1+z)^{2}\right]^{1 / 2} \mathrm{~d} t$ and values for $H_{0}, \Omega_{M}$ and $\Omega_{\Lambda}$ taken from WMAP (Peiris et al. 2003). Thus, at $z=0.2$, $\delta z \approx 3 \times 10^{4} c \delta t$.

\section{Application to 1ES 1101-232}

The SED of the attenuating EBL can be deconvolved from $\gamma$-ray observations of extragalactic sources (TeV blazars), given a priori knowledge on the intrinsic spectra. Modelling observed spectra as power-laws, the effect of PP is to soften the intrinsic spectral index, increasingly so with EBL intensity. Hence, using observations of the farthest $\mathrm{TeV}$ blazar and assuming the hardest possible intrinsic spectrum puts an upper limit on the 


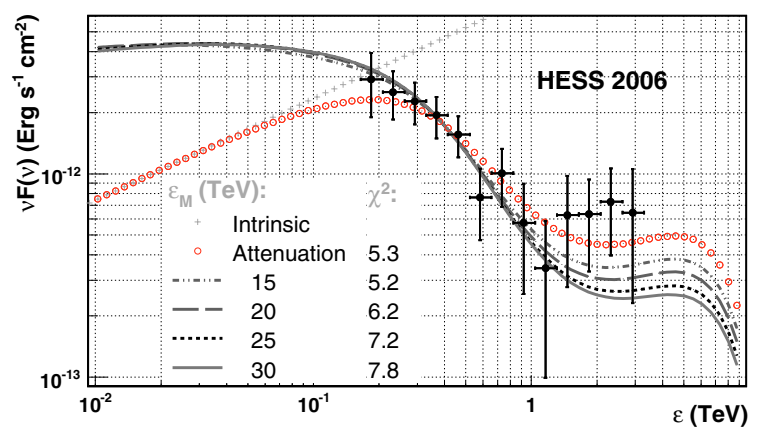

Fig. 3. 1ES 1101-232 observed and modelled spectra with a maximal (HESS 2006) EBL and including cascades with no magnetic field. HESS observations points are in black (Aharonian et al. 2006). Markers indicate the attenuation only observed spectrum (circles) and the corresponding intrinsic spectrum (crosses), whereas the lines indicate the observed spectra with cascade emissions. Intrinsic spectra are in the form of $v F_{v} \propto E^{0.5} \mathrm{~d} v$ and adjusted to the data. Cascade emission accumulates at $100 \mathrm{GeV}$ and below, softening the spectra compared to pure absorption. The HESS upper limit on the EBL remains valid after taking the full emission from cascades into account.

EBL responsible for attenuation. Current theoretical understanding of shock acceleration limits the intrinsic particle distribution in blazars to a power-law of index no harder than a 1.5 and correspondingly, an intrinsic photon spectrum $\mathrm{d} N \propto E^{-\Gamma} \mathrm{d} E$ with $\Gamma \geq 1.5$ (Aharonian et al. 2006).

1ES 1101-232, at $z=0.186$, is currently the farthest known $\mathrm{TeV}$ source and was used by the HESS collaboration to set an upper limit to the EBL corresponding to the HESS 2006 SED shown in Fig. 1. The comparison between a $\Gamma=1.5$ power-law attenuated by the HESS $2006 \mathrm{EBL}$ (without cascade, $c_{B}=0$ ) and the data is shown in Fig. 3, reproducing the results of Aharonian et al. (2006). Attenuated spectra taking into account the full cascade emission with $c_{B}=1$ (i.e. a null EMF) are also shown for various values of the maximum energy $\epsilon_{M}$ to which the intrinsic power-law extends. Since cascades initiated at higher energies increase the photon populations in lower ones, one might expect the final spectra to appear harder than for pure absorption. However, because IC occurs predominantly on the CMB, the cascade emission accumulates below $100 \mathrm{GeV}$, softening the spectrum between $100 \mathrm{GeV}$ and $1 \mathrm{TeV}$. High values of $\epsilon_{\mathrm{M}}$ lead to more cascading and more softening. The $\chi^{2}$ values suggest $\epsilon_{\mathrm{M}}<15 \mathrm{TeV}$, although further observations, particularly above $1 \mathrm{TeV}$, would be necessary in order to confirm this. For such low $\epsilon_{M}$ values, not many photons initiate cascades. For higher $\epsilon_{M}$, the softening is such that a lower EBL would be needed to match the data. Thus the HESS 2006 upper limit found by Aharonian et al. (2006) holds strong, even in this extreme limit where all the cascade emission is received by the observer.

Inversely, the intrinsic $\gamma$-ray spectrum at the source can be obtained given some assumption on the intervening EBL. Using the lower limit on the EBL set by galaxy counts (Primack 2005 in Fig. 1) gives a limit on how soft the intrinsic spectrum can be. For pure absorption, the best fit has $\Gamma=1.95 \pm 0.19$ (Fig. 4). As expected, this is softer than the $\Gamma=1.5$ assumed above, yet still suggests that a good fraction of the $\gamma$-ray energy in 1ES 1101-232 is output above a TeV. A hard $\Gamma \leq 2$ intrinsic spectrum is needed if cascade emission is to contribute significantly to the low-energy continuum (Aharonian et al. 2002). 1ES 1101-232 is the first blazar where the intrinsic spectrum is constrained to be hard enough for this, even in the minimal EBL limit.

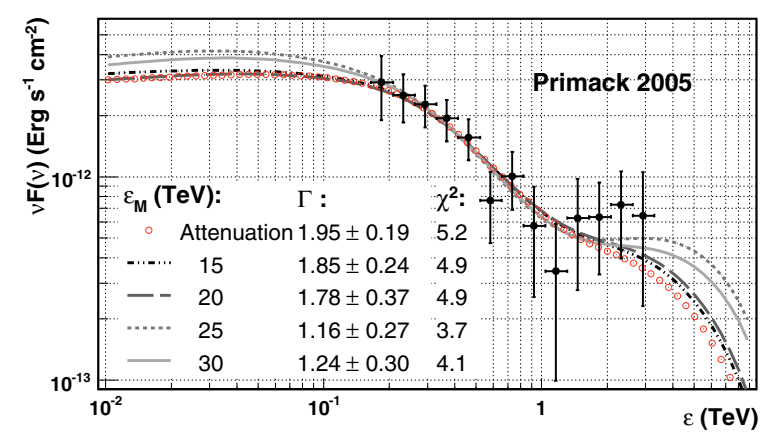

Fig. 4. 1ES 1101-232 observed and modelled spectra with a minimal (Primack 2005) EBL and including cascades with no magnetic field. The intrinsic spectrum is now adjusted to the HESS data leaving the spectral index $\Gamma$ free. For pure absorption, the best index is $\Gamma=1.95$. With cascades, the index hardens as IC emission softens the propagated spectrum. For high $\epsilon_{\mathrm{M}}$, the best index softens again so as to limit the amount of cascading but the fit worsens. Significant cascading on the minimal EBL and in a very weak EMF implies a very hard input spectrum in order to account for the observations.

Including cascade emission in the fit (Fig. 4) hardens even more the intrinsic spectrum as the cutoff $\epsilon_{\mathrm{M}}$ increases and cascades contribute more and more to the observed spectrum. For higher $\epsilon_{\mathrm{M}}$, the best fit $\Gamma$ increases again to mitigate the pronounced softening from the strong cascading but the fit worsens. This also holds for (implausibly) high values of $\epsilon_{\mathrm{M}}>100 \mathrm{TeV}$, for which cascade emission largely dominates at a few $\mathrm{TeV}$. The hard intrinsic spectra found here, assuming the Primack 2005 is indeed the minimum possible EBL, suggest either that $\epsilon_{M}$ is not greater than a few $\mathrm{TeV}$, so that there is little cascade emission in the $\mathrm{TeV}$ range, or that a large part of the cascade emission is lost due to diffusion on the EMF.

As discussed in Sect. 2, the electron diffusion on the EMF depends on the ratio $R_{\mathrm{L}} / C_{\mathrm{IC}}$. The effect on the observed spectra is now taken into account by setting $c_{B}=0$ when $R_{\mathrm{L}} / C_{\mathrm{IC}}<$ 300 (corresponding to a maximum deviation on the line-ofsight of $0.1^{\circ}-0.2^{\circ}$ equal to the best GLAST angular resolution) and $c_{B}=1$ otherwise. For example, an EMF of $10^{-6} \mathrm{nG}$ means that emission from electrons of energy $E \lesssim 20 \mathrm{TeV}$ is suppressed. This will lead to low-energy cutoff in the cascade spectrum as only emission from pairs above a certain energy reaches the observer. The overall spectrum appears as a hump between $\gamma^{2} h v_{\mathrm{CMB}}$ (with $\gamma$ the Lorentz factor of the electrons for which $R_{\mathrm{L}}=300 C_{\mathrm{IC}}$ ) and $100 \mathrm{GeV}$ (above which absorption dominates). Hence, a non-zero EMF leads to a reduction of the overall cascade emission seen by the observer (compared to Figs. 3-4) but can also lead to a well-defined signature above the continuum.

Figure 5 shows the observed spectra for a Primack 2005 EBL and for EMF intensities between $10^{-9}$ and $10^{-6} \mathrm{nG}$. The intrinsic power-law index was left free but its cutoff $\epsilon_{\mathrm{M}}$ was fixed at either $10 \mathrm{TeV}$ or $20 \mathrm{TeV}$. The best fit index $\Gamma$ is then found for each value of the EMF. In both cases, the spectra for an EMF $\gtrsim 10^{-6} \mathrm{nG}$ are not much different from the pure absorption case as most of the cascade emission is isotropised and lost to the observer. With $\epsilon_{\mathrm{M}}=10 \mathrm{TeV}$, the best-fit intrinsic slopes are flat in $v F_{v}$ and the cascade emission is essentially indistinguishable from the $\mathrm{GeV}$ continuum for any value of the EMF. The intrinsic emission is assumed here to be a simple power-law over the whole energy range. More realistic modelling would result in a curved intrinsic Compton component. The cascade emission 

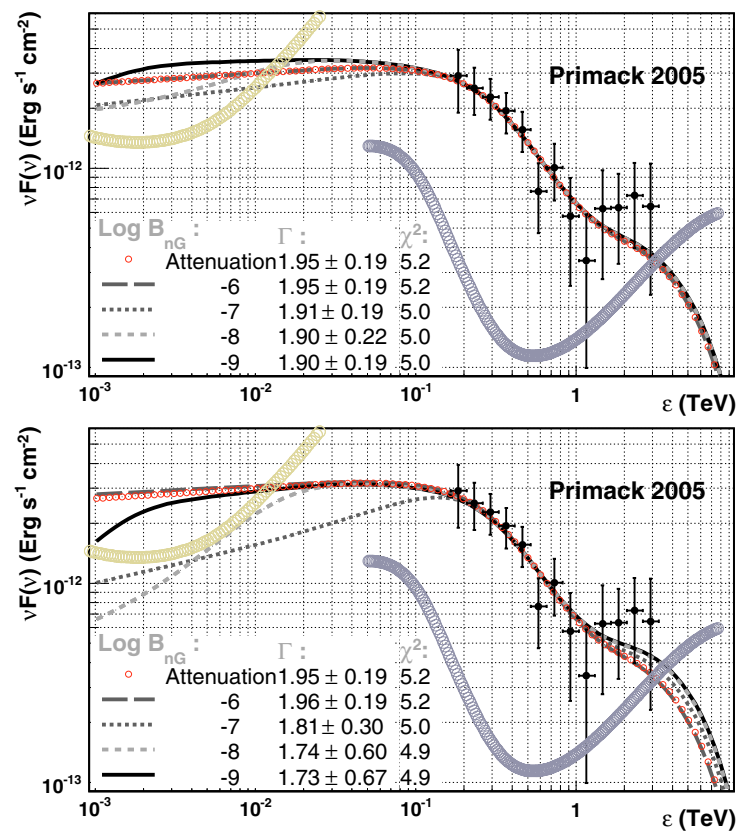

Fig. 5. Observed spectra for the Primack 2005 EBL and various EMFs between $10^{-9}$ and $10^{-6} \mathrm{nG}$. The spectra are adjusted to the HESS points, leaving $\Gamma$ free but fixing $\epsilon_{\mathrm{M}}=10 \mathrm{TeV}$ (top) or $20 \mathrm{TeV}$ (bottom). For high EMFs the cascade emission does not reach the observer and the spectrum is identical to the pure absorption case. For very low EMFs the results are similar to those shown in Fig. 4. Intermediate values lead to a more or less pronounced bump at $1-100 \mathrm{GeV}$ energies over the intrinsic continuum. Hatches represent projected 5- $\sigma$ sensibilities for one year of observation with GLAST $(\leq 30 \mathrm{GeV})$ and $50 \mathrm{~h}$ with HESS-2 $(\geq 50 \mathrm{GeV})$.

might then be more readily identifiable over an intrinsic continuum rising from $\mathrm{GeV}$ to $\mathrm{TeV}$ energies.

Stronger cascading, as a result of a higher cutoff energy $\epsilon_{\mathrm{M}}$ and/or a higher EBL density, makes the hump apparent for the same reason. The intrinsic spectrum is then necessarily much harder, enabling the contribution from the cascade to stand out over the continuum. The bottom panel of Fig. 5 shows that EMF intensities of $10^{-9}-10^{-8} \mathrm{nG}$ can be identified using GLAST and HESS-2 if $\epsilon_{\mathrm{M}}=20 \mathrm{TeV}$. Cascade emission is not diluted for EMF intensities weaker than $10^{-9} \mathrm{nG}$ and there is no spectral feature to measure the EMF. Surprisingly, in most cases 1ES 1101-232 is only slightly above the GLAST oneyear detection limit. Unless they become active and flaring, low flux state blazars detected by HESS such as 1ES 1101-232 are likely to be difficult to detect with GLAST, illustrating the advantage provided by the large collecting area of ground-based Cherenkov arrays (but at higher energy thresholds). Similar results are obtained by keeping $\epsilon_{\mathrm{M}}$ at $10 \mathrm{TeV}$ but using the stronger HESS 2006 EBL. However, in this case, the fitted intrinsic slopes are very hard $(\Gamma \approx 1.1)$ when the EMF intensities are lower than $10^{-7} \mathrm{nG}$.

The softest values of $\Gamma$, which are the most plausible given the present knowledge on blazars, favour values of the EMF higher than $10^{-6} \mathrm{nG}$ and/or a cutoff energy below $20 \mathrm{TeV}$. VHE emission from nearby, little-attenuated blazars can be investigated for evidence of cutoffs at energies $>20 \mathrm{TeV}-$ although it should be noted that e.g. HESS observations of Mkn 421 $(z=0.03)$ taken at a high flux actually measure an exponential cutoff at $3 \mathrm{TeV}$ (Aharonian et al. 2005). EMF intensities $\gtrsim 10^{-6} \mathrm{nG}$ are consistent with measures inside clusters and superclusters. Such structures may reach $10-50 \mathrm{Mpc}$ in size, which is greater than the attenuation length for $\gamma$-rays above $50 \mathrm{TeV}$. Furthermore, the largest voids, where the EMF is expected to be very small, have a size of $20 \mathrm{~h}^{-1} \mathrm{Mpc}$ (Patiri et al. 2006), smaller than the distance to 1ES 1101-232. Hence, cascades are likely to be initiated inside walls. As $C_{\mathrm{IC}}$ is only of the order of $1 \mathrm{Mpc}$, such cascades reemit most of their energy within the confines of the clusters, and thus are subject to diffusion. In this case, the cascade emission can only be detected by resolving the faint halo surrounding the $\gamma$-ray source.

\section{Conclusion}

The impact of extragalactic cascade emissions on the GeV-TeV spectrum of 1ES 1101-232 has been investigated and shown to soften the observed spectrum in the TeV range compared to pure absorption. This occurs because most of the cascade emissions occurs at $100 \mathrm{GeV}$ and below. As a result, the upper limits on the EBL determined by HESS are strengthened in the sense that taking cascades into account would lead to harder intrinsic spectra than judged plausible, or to a reduced EBL upper limit. Inversely, using lower limits on the EBL coming from galaxy counts, the intrinsic spectrum of 1ES 1101-232 is found to have $\Gamma \leq 1.95$, with very hard values if there is an important contribution from cascade emission. This is at odds with current theoretical and observational understanding of blazars. A cutoff $\lesssim 10 \mathrm{TeV}$ in the intrinsic spectrum would limit the cascade contribution. This contribution would also be quenched if the EMF intensity is greater than $10^{-6} \mathrm{nG}$, as expected away from voids. A lower EMF increases the amount of cascade emission reaching the observer in the $\mathrm{GeV}$ band, with a signature in the GLAST band for intensities $\sim 10^{-8} \mathrm{nG}$ - but at the price of a hard intrinsic spectrum so as to fit the HESS observations.

\section{References}

Aharonian, F. A., Coppi, P. S., \& Völk, H.J. 1994, ApJ, 423, L5

Aharonian, F. A., Timokhin, A. N., \& Plyasheshnikov, A. V. 2002, A\&A, 384, 834

Aharonian, F., Akhperjanian, A. G., Aye, K.-M., et al. 2005, A\&A, 437, 95

Aharonian, F., Akhperjanian, A. G., Bazer-Bachi, A. R., et al. 2006, Nature, 440, 1018

Biller, S. D. 1995, Astropart. Phys., 3, 385

Cheng, L. X., \& Cheng, K. S. 1996, ApJ, 459, L79

Dai, Z. G., Zhang, B., Gou, L. J., Mészáros, P., \& Waxman, E. 2002, ApJ, 580, L7

Dole, H., Lagache, G., Puget, J.-L., et al. 2006, A\&A, 446

Fan, Y. Z., Dai, Z. G., \& Wei, D. M. 2003, A\&A, 13

Gould, R. J., \& Schréder, G. 1966, Phys. Rev. Lett., 16, 252

Jones, F. C. 1967, Phys. Rev., 167, 1159

Kronberg, P. P. 1994, Reports of Progress in Physics, 57, 325

Lagache, G., Dole, H., \& Puget, J.-L. 2003, MNRAS, 338, 555

Neronov, A., \& Semikoz, D. V. 2006, ArXiv Astrophysics e-prints

Patiri, S. G., Betancort-Rijo, J. E., Prada, F., Klypin, A., \& Gottlöber, S. 2006, MNRAS, 369, 335

Peiris, H. V., Komatsu, E., Verde, L., et al. 2003, ApJS, 148, 213

Plaga, R. 1995, Nature, 374, 430

Primack, J. 2002, in COSPAR, Plenary Meeting

Primack, J. R., Bullock, J. S., Somerville, R. S., \& MacMinn, D. 1999, Astropart. Phys., 11, 93

Primack, J. R., Bullock, J. S., \& Somerville, R. S. 2005, in High Energy GammaRay Astronomy, ed. F. A. Aharonian, H. J. Völk, \& D. Horns, AIP Conf. Proc, 745,23

Protheroe, R. J. 1986, MNRAS, 221, 769

Protheroe, R. J., \& Stanev, T. 1993, MNRAS, 264, 191

Stecker, F. W., de Jager, O. C., \& Salamon, M. H. 1992, ApJ, 390, L49

Vallée, J. P. 2004, New A. Rev., 48, 763

Widrow, L. M. 2002, Rev. Mod. Phys., 74, 775

Xu, C., Lonsdale, C. J., Shupe, D. L., O’Linger, J., \& Masci, F. 2001, ApJ, 562, 179

Zdziarski, A. A. 1988, ApJ, 335, 786 